\title{
Antioxidant Modulation of Hematological Toxicity during Chemotherapy for Breast Cancer
}

Hojouj Mohammad*, Bondarenko I, Zavizion V, Artemnko M, Soloviova N, Bondarenko Y, Dmytrenko K and Urzhumova N

Department of Oncology and Medical Radiology, Dnepropetrovsk Medical Academy, 31, Blyzhnya Str, Dnepropetrovsk, 49102, Ukraine

\begin{abstract}
Side effects of anticancer drugs seriously limit the achievement of the maximum therapeutic effect of the most cytostatic. One of the pathophysiological bases of side effects is the ability of cytotoxic agents to intensify the freeradical processes and the consequent lipid peroxidation (LPO) in the cell membranes of various organs. When the antitumor treatment is conducted, the antioxidant enzyme deficiency increases, there is the depletion of nonenzymatic and enzymatic mechanisms of oxidation protection units, resulting in a reduction of organism resistance and damage to vital organs and systems. In this connection it is important to study the possibility of correction of violations occurring in cancer patients with drugs with antioxidant action type.
\end{abstract}

Keywords: Cytostatic; Molecular endocrine therapy; Oxidative stress; Tumor cells; Immunohistochemical

\section{Introduction}

\section{Objectives of the Study}

1. To investigate the condition of antioxidant system in the blood of oncological patients during chemotherapy for breast cancer;

2. To study the efficacy of antioxidant therapy in the prevention of the development of leukopenia, as well as the possibility of improving treatment outcomes in their use;

3. To justify the use of antioxidant therapy for the prevention of hematological toxicity of chemotherapy according to levels of biochemical markers of the connected systems of antioxidant protection and oxidative stress.

\section{Case Study}

\section{Individualize treatment of hormone-dependent breast cancer}

Around 13500 female is diagnosed with breast cancer in Ukraine. System control in both local and recurrent breast cancer is topical issue and should be thoroughly discussed considering economic situation in country.

Standard drug treatment regimen does not always give positive results. Practically speaking, physicians use standard treatment regimen based upon tumor IHC analysis, if any.

Immunohistochemical study of breast cancer biological markers has two endpoints: 1) identify high risk groups for progression in early stage disease; additional examination and Treatment is required; 2) evaluation of individual responsiveness to planned or received treatment. Surrogate molecular classification is used to identify luminal subtypes, Her2-positive, basal and rare types. Luminal subtypes includes subtype A, about $40 \%$ of all breast cancer, this subtype characterized by positive estrogens (ER), progesterone (PR), absence of growth factor hyper fixation (Her2/neu) and low level of Ki67 proliferation $(<20 \%)$. Luminal subtype B is subdivided into Her2positive and Her2-negative subtypes. Luminal Her2-negative subtype differs from luminal A subtype by higher or high level of Ki57 marker ( $\geq 20 \%$ ). Luminal B Her2-positive subtype expresses Her/neu, and has high level of Ki67 marker. Cases where Her2/neu has low level of proliferation $($ Ki67<20\%) should be analyzed separately [1-7].
Classic example of breast cancer individualization treatment is by determining of reproductive hormones and epidermal growth factor [8]. Prognostic value of ER and PR determination for endocrine therapy is confirmed by meta-analysis of 55 randomized trials with 37000 breast cancer patients. It is proved that ER expression in breast tumor indicates potential responsiveness to drug treatment, this is aimed to remove estrogens source and its effect recovering. PR is the first step in cell reaction to progestines and it determines cell responsiveness to some drugs, but mainly because it synthesis in breast cancer cells is prompt by estrogens. Presence of PR confirms ER functional activity. Breast cancer patients who has both or at least one of steroid hormones receptors have more favorable prognosis than those has no receptors. Thus $75 \%$ of patients with both positive receptors responses effectively to endocrine therapy and 50\% with only ER positive receptor. However there was response to endocrine therapy in $10 \%$ of patient with both negative receptors [9].

A high level of estrogen receptors is associated, in front of all, with an increase in overall and not relapse-free survival, and is a predictor of the effectiveness of hormone therapy. In recent years Ki-67 proliferation index has been used to predict the effectiveness of treatment [10]. The panel of estrogen progestin receptor markers, Her2/neu and Ki-67, is today standard both at the stage of primary diagnosis and in morphological studies in the course of treatment. Taking into account these four markers, the main immunomorphologic subtypes of breast cancer are listed above. In the case of neoadjuvant treatment or treatment of metastatic cancer (if there is a possibility of re-biopsy in the dynamics), Ki-67 has recently been used as a marker for the effectiveness of treatment. Repeated biopsy is performed after 3 weeks of treatment. Decrease of marker level is the first morphological

*Corresponding author: Mohammad $\mathrm{H}$, Associate Professor Department of Oncology and Medical Radiology, Dnepropetrovsk Medical Academy, 31, Blyzhnya Str., Dnepropetrovsk, 49102, Ukraine, Tel: +380567560399/+380675673277; Fax: 380563777797; E-mail: hojouj@yahoo.com

Received September 05, 2017; Accepted October 06, 2017; Published October 16, 2017

Citation: Mohammad H, Bondarenko I, Zavizion V, Artemnko M, Soloviova N, et al (2017) Antioxidant Modulation of Hematological Toxicity during Chemotherapy for Breast Cancer. J Cytol Histol 8: 478. doi: 10.4172/2157-7099.1000478

Copyright: (c) 2017 Mohammad H, et al. This is an open-access article distributed under the terms of the Creative Commons Attribution License, which permits unrestricted use, distribution, and reproduction in any medium, provided the original author and source are credited. 
predictor of treatment efficacy, not only of hormone, but also of chemotherapy. Conversely, an increase in marker level is a predictor of an unfavorable forecast [11-15].

So, consider the information regarding the most favorable subtype - luminal A, and more aggressive, but still hormone-dependent luminal B.

As long experience shows, the highest effectiveness of anti-estrogen therapy is observed, provided that the tumor expresses both estrogen receptors and progesterone receptors. The most commonly used drugs for anti-estrogen therapy are tamoxifen and toremifene. Preparations of choice include aromatase inhibitors (non-steroidal letrozole, anastrozole and steroid exemestan) and a selective inactivates of estrogen fulvestrant receptors. However, 10-15 percent of ER-positive tumors do not respond to tamoxifen, on the contrary, tumor growth may be noted [16-18].

The adverse effect of tamoxifen on the progression of breast cancer is strongly correlated with the amplification of the cyclin D1 oncogene. Blocking cyclones helps to eliminate proliferation, caused by estrogens and reduces the activity of estrogen receptors. Expression of cyclins activates extra genomic action of estrogens through stimulation of tyrosine kinase receptors or a self-assembly of functioning protein complexes in the cell's cytoplasm. These complexes include in the cascade other signaling molecules that activate transcription. Thus, with high expression of cyclin D1, anti-estrogen blocking of estrogen receptors will be less effective and the proliferation of tumor cells will be either due to the agonistic effects of tamoxifen or estrogen action of estrogens. Patients who have an enlarged copy of this gene should receive aromatase inhibitors as hormones, or abstain from the use of antagonists of the estrogen signaling cascade [19].

It should be taken into account that the negative or low level (up to $30 \%$ ) of the expression of cyclin D1 is such as to promote tamoxifen. If the level of expression of cyclin D1 is medium to high, the action of tamoxifen is either agonistic or lost and triggers the mechanism of estrogen extracorporeal action. Thus, according to Skvortsov and Manichas Patients with a negative or low expression level of cyclin D1 had no recurrence of breast cancer during the five years of adjuvant tamoxifen, and in most patients with moderate to high expression, progression was recorded, mainly bone metastases $(62 \%$ of patients with average level of expression and in $68 \%$ - with high). Defeat of soft tissues in the postoperative scar area occurred in $14 \%$ of patients with mean expression level and $28 \%$ with high expression of cyclin D1. Metastases in the lungs and $4 \%$ with high levels of soft tissue metastases occurred in $4 \%$ of patients with average levels of expression. Considering that even in advanced and super-radical operations, 70$80 \%$ of breast cancer patients who have not undergone additional treatment progress sooner or later [18], it is not difficult to conclude that the average and high levels of expression Cyclin D1 presumably causes tumor resistance to selective estrogen receptor modulators [20-22].

One of the markers of sensitivity to hormone therapy, some authors consider the regulator of apoptosis Bcl-2. Having studied the experience of many researchers, Baryshnikov and Stepanova argues that $\mathrm{Bcl}-2$ can predict the efficacy of adjuvant therapy with tamoxifen in patients with a hormone susceptible breast cancer from damage to regional lymph nodes, especially in the group of ER negative tumors [20].

Other factors influencing the effectiveness of antagonists of estrogens are the estrogen receptor co-activator AIB1 and Her2/neu. The estrogen receptor co-activator AIB1 (amplified in breast 1) is also referred to as SRC-3 (steroid receptor coactivator-3), NCOA3 (nuclear receptor coactivator 3) RFC3 (Replication factor C subunit 3). AIB1, like the estrogen receptor itself, is phosphorylated and thus functionally activates MARK (mitogen-activated protein kinase); thus, high levels of activated AIB1 may reduce the antagonistic effects of tamoxifen, especially in tumors that also overexpress HER/2. Thus, the expression of AIB1 is an important prognostic marker indicative of tamoxifen resistance. In this case, tumors expressing AIB1 remain susceptible to aromatase inhibitors [23,24].

Sensitivity data for Her2/neu positive tumors to tamoxifen are ambiguous. Herx/neu-expressing tumors are more likely to exhibit tamoxifen resistance than aromatase inhibitors. Thus, in study 024 , "Lethrozol versus tamoxifen in neoadjuvant breast cancer therapy", the objective response of c-crbB-positive tumors to letrozole therapy was in $88 \%$ of patients, while for tamoxifen alone, $21 \%$ of patients $(\mathrm{p}=0.0004)$ [25]. The high effectiveness of aromatase inhibitors is also reported by Li Zhu. The analysis of the key findings of multicenter international clinical studies suggests the anticipated resistance of Her2/neu positive tumors to tamoxifen, but not to aromatase inhibitors. Later, the correctness of these findings was confirmed by clinical trial BIG 1-98 (80,010 patients were trailed). As one of the possible mechanisms for the development of estrogen receptor resistance of positive Her2/neu positive tumors, an adaptive increase in Her2/neu levels in response to long-term endocrine effects is considered. Thus, on the model of breast cancer cells with enormous resistance due to prolonged exposure to tamoxifen, it has been shown that the use of a double inhibitor of the tyrosine kinase receptor of the epidermal growth factor (lapatinib) leads to a restoration of sensitivity to tamoxifen. This effect is related to the genomic mechanism of action of estrogen receptors due to inhibition of mutual activation of EP and Her2. In any case, Her2/neu positive tumors are mostly tamoxifen resistant. However, they are quite often susceptible to aromatase inhibitors, and the addition of trastuzumab or lapatinib to therapy results in a significant improvement in the treatment outcome in patients with luminal Her2/neu positive breast cancer.

Another marker of tamoxifen resistance to hormone-dependent breast cancer is the mutation of the $\mathrm{p} 53$ gene. A mutation occurs in 10$20 \%$ of cases of luminal A and 13 to $31 \%$ of luminal B cell cancer [26] and is a marker of an unfavorable prognosis. Initially, the tamoxifen resistance of p53-mutated cells was shown in vitro on MCF-7 cell lines of breast cancer [27]. Subsequently, these data were confirmed in the meta-analysis of 4683 patients receiving tamoxifen after adjuvant chemotherapy.

The mechanism of resistance to aromatase inhibitors remains poorly understood. One of the probable mechanisms of acquired resistance to this group of drugs is the ectopic overexpression of aromatase by cancer cells. Another hypothesis - as a result of the long term absent estrogen aromatase inhibitors simulated the effect of the blockade of estrogen synthesis This mechanism of resistance is implemented through the PI3K/Akt/mTOR signaling pathway, To overcome the acquired resistance allows the use of mTOR inhibitors, the effectiveness of which is proven by clinical studies TAMRAD (tamoxifen+avorelimus), low level of progestin receptors, and their loss in the treatment process (regardless of the level of estrogen receptors) indicates a questionable sensitivity to inhibitors a romatasi [28].

In practice, the probability of such a mechanism of primary resistance is indicated by the presence of a metabolic syndrome in the patient, manifested before all in the increase in body weight $\left(\mathrm{BMI}>25 \mathrm{~kg} / \mathrm{m}^{2}\right.$ ) with the tendency or development of obesity before all by the abdominal type. The use of metformin (dimethylbiguanide) 
in such patients helps to avoid the effect of primary resistance to aromatase inhibitors. Little is known about the molecular targets of the proapoptotic action of COX-2 inhibitors, although in a number of cases in apoptotic cells a violation of phosphorylation of kinase Akt (Protein kinase B alpha) has been noted [29].

- The HIF-1a/VEGF signaling pathway (induced by hypoxia factor 1) plays an important role in the development of resistance to aromatase inhibitors. There is a direct correlation between the expression of HIF-1 $\alpha$ tumor cells and the resistance to neoadjuvant therapy with aromatase inhibitor (letrozole). Works that have a direct evaluation of HIF-1 $\alpha$ are few.

- The key target of HIF-1a - vascular endothelial growth factor (VEGF) has been studied well. Estradiol stimulates the synthesis of VEGF, and the action of HIF-1a and ERa in relation to VEGF is synergistic.

- The development of hormone resistance in breast cancer is accompanied by activation of the VEGF/VEGFR2/MAPK signaling pathway.

- Celecoxib (a specific VEGF inhibitor) affects the activity of the VEGF promoter and directly inhibits the synthesis of VEGF, in particular avoiding unwanted effects of tamoxifen

Thus, in the case of breast cancer diagnosis with the $\mathrm{EP}+\mathrm{PR}+\mathrm{Her} 2 /$ neu+++ phenotype, it is mandatory to study all markers Ki67, cyclin D1, AI.

The chemo-sensitivity of the tumor is appreciably correlated with the expression characteristics of certain molecules. Currently, molecular markers of fluoropyrimidine treatment efficacy are most studied: DPD, TS, TP TS (thymidylatesintetasa), a key phosphatedependent mimetic enzyme in the process of DNA synthesis that catalyzes the formation of thymidylate, a precursor of thymidine triphosphate, a nucleotide necessary for the synthesis of DNA - and is the main target for the effect of uracil and 5 fluorouracil ( 5 active urethyl antimetabolites). The active metabolite of 5FU, 5-fluoro-2deoxyuridine monophosphate (FdUMP), interacts with TS, resulting in blockage of DNA synthesis. The excess of the TSF $5 \mathrm{~F}$ target leads to the fact that the transferable doses of the drug cannot "saturate" this enzyme and prove to be inevitably ineffective [30,31].

\section{Materials and Methods}

The study of hematological parameters was evaluated in two groups of patients - control group (60 patients) and the study group (50 patients) with study drug. Both groups of patients received chemotherapy for breast cancer with taxanes+anthracyclines. In the period between courses of study group patients received antioxidant therapy. We studied the incidence of leucopenia during treatment. Also markers of protein oxidative modification, system of nitrogen oxide, thiol system and apoptotic markers were studied.

\section{Results and Discussion}

1) After prophylactic administration of antioxidant drugs in the study group it was recorded a 2.05-fold greater level of thyol groups than in the control group of patients $(\mathrm{p}<0.05)$. In the study of spontaneous and stimulated markers of oxidative modification of the protein as a marker of oxidative stress, it was revealed that antioxidant therapy is effective protector of cell membranes from oxidative stress. Patient who received antioxidant drugs have 1, 27-77 times lower level of enzymes adelhydfenihydrazon and ketonefenihydrazon comparing to the control group [32-35].

2) In the study group of patients who received antioxidant therapy, lower incidence of leukopenia $(66.7 \%$ vs. $46.0 \%)(\mathrm{p}<0.05)$ by reducing the incidence of deep leucopenia was observed. Indicators of anemia and thrombocytopenia occurring were not statistically different.

3) Reduction in the activity of antioxidant enzymes of glutathione level and the displacement of the thiol-disulfide equilibrium in the direction of reducing of the content of reduced forms indicates a reliable clinical need for the personal use of drugs with glutathioneprotective effect and antioxidant potential.

\section{Conclusion}

According to the results of a randomized clinical trial of PACS01, the addition of docetaxel to epirubicin and 5-fluorouracil as adjuvant chemotherapy in a group of patients with estrogen-positive tumors and a high Ki-67 index is advisable. These results were confirmed in the Cancer International Research Group 001 trial. However, these findings are not consistent with International Breast Cancer Study Group Trials VIII and IX. In these studies, the predictive role of the high level of Ki67 expression in the group with receptor-positive breast cancer without signs of disease in the lymph nodes in relation to adjuvant therapy with the inclusion of methotrexate, cyclophosphamide and 5-fluorouracil in addition to endocrine therapy was demonstrated. Thus, it is important to conduct studies aimed at isolating groups of patients with high Ki-67 rates who can maximize the benefit of various adjuvant chemotherapy regimens.

\section{References}

1. NCCN Guidelines Version 2.2016 Invasive Breast Cancer. BINV-2, Recent Updates to NCCN Clinical Practice Guidelines in Oncology (NCCN Guidelines ${ }^{\circledR}$ ).

2. Dengin NV, Rodionov VV (2010) Radiation therapy of operable breast cancer after radical mastectomy: what, where, when Practical Oncology 11: 221-227.

3. Kosse VA, Bezruk NG, Ovsyannikov PV, Zavizion VF (1999) Recurrences of breast cancer after organ-preserving treatment. Bulletin of Problems of Biology and Medicine 1: 119-122.

4. Bolotina LV, Zakiryakhodzhaev AD, Malygin SE, Medvedev SV, Pak DD, et al (2016) Clinical Recommendations For Prevention, Diagnostics And Treatment Of Patients With Breast Cancer "Printing Center" Luck 47.

5. Tyulyandin SA, Nosov DA, Perevodchikova NI (2010) ESMO [Minimum Clinical Recommendations of the European Communication on Medicine Oncology (ESMO) Editors of the Russian translation Moscow: Publishing Group of RONTS them. Blokhin NN, Russian Academy of Medical Sciences 436.

6. Kushlinskii NE, Timofeev YS, Solov'ev YN, Gerstein ES, Lyubimova NV, et al. (2014) Components of the RANK/RANKL/OPG system, IL-6, IL-8, IL-16, MMP-2, and calcitonin in the sera of patients with bone tumors. Bull Exp Biol Med 157: 520-523.

7. Perevodchikova NI, Steninova MB (2014) Guidelines for chemotherapy of neoplastic diseases. Moscow: Practice.

8. Toffoli G, Russo A, Innocenti F, Corona G, Tumolo S, et al. (2003) Effect of methylenetetrahydrofolate reductase 677CT polymorphism on toxicity and homocysteine plasma level after chronic methotrexate treatment of ovarian cancer patients. Int J Cancer 103: 294-299.

9. Novik AV, Zhabin AS, Importancia LA (2011) Biomarkers for diagnostics and determination of tactics of treatment of patients with tumors with unconditional primary localization. Practical oncology 12: 178-184.

10. Dobrenky MN, Dobrenky AM (209) Molecular-biological and biochemical predictors of breast cancer (review of literature).

11. Lazukin AV (2013) The role of the Ki-67 marker in predicting breast cancer. RNM.

12. Cuzick J, Dowsett M, Wale C (2009) Prognostic value of a combined ER, PgR, Ki67, HER2 immunohistochemical (IHC4) score and the comparison with the $\mathrm{GHI}$ recurrence score-results from TransATAC. Cancer Res. 69: 503. 
Citation: Mohammad H, Bondarenko I, Zavizion V, Artemnko M, Soloviova N, et al. (2017) Antioxidant Modulation of Hematological Toxicity during Chemotherapy for Breast Cancer. J Cytol Histol 8: 478. doi: 10.4172/2157-7099.1000478

13. Penault-Llorca F, Andre F, Sagan C (2009) Ki67 expression and docetaxel efficacy in patients with estrogen receptor-positive breast cancer. J Clin Oncol 27: $2809-2815$

14. Dowsett M, Smith IE, Ebbs SR (2005) Short-term changes in Ki-67 during neoadjuvant treatment of primary breast cancer with anastrozole or tamoxifen alone or combined correlate with recurrence-free survival. Clin Cancer Res 11: 951-958.

15. Leo A, Cardoso F, Durbecq V (2002) Predictive molecular markers in the adjuvant therapy of breast cancer: state of the art in the year 2002. Int J Clin Oncol 7: 245-253.

16. Zakharov Yu A (2012) Treatment and prevention of metastatic cancer. TNM Kherson 400.

17. Kryachok IA, Gubareva AA, Aleksik EM, Filonenko ES (2013) The Role Of Adjuvant Chemotherapy In Treatment Of Numbly-Cellular Lung Cancer. Clinical oncology 2: 118-122.

18. Wilcken NR, Prall OW, Musgrove EA, Sutherland RL (1997) Inducible overexpression of cyclin D1 in breast cancer cells reverses the growth-inhibitory effects of antiestrogens// Clin Cancer Res 3: 849-854.

19. Jirstrom K, Stendahl M, Ryden L (2005) Adverse effect of adjuvant tamoxifen in premenopausal breast cancer with cyclin D1 gene amplification. Cancer. Res 65: 8009-8016

20. Kryachok IA, Gubareva AA, Alexik EM, Filonenko ES (2013) Modern principles of adjuvant treatment in malignant neoplasms of the thorax Clinical oncology 2: $118-122$.

21. Layla J. Al-Mansouri, Majed S. Alokail (2006) Molecular basis of breast cancer. Saudi Med J Vol. 27: 9-16.

22. Osborne KC, Bardou V, Hopp TA, Chamness GC, Susan G (2003) Role of the Estrogen Receptor Coactivator AIB1 (SRC-3) and HER-2/neu in Tamoxifen Resistance in Breast Cancer. Journal of the National Cancer Institute 95: 353-361.

23. Font de Mora J, Brown M (2000) AIB1 is a conduit for kinase-mediated growth factor signaling to the estrogen receptor. Mol Cell Biol 20: 5041-5047.

24. De Placido S, De Laurentiis M, Carlomagno C (2003) Twenty-year results of the Naples GUN randomized trial: Predictive factors of adjuvant tamoxifen efficacy in early breast cancer. Clin Cancer Res 9: 1039-1046.

25. Elledge RM, Green S, Ciocca D (1998) Her-2 expression and response to tamoxifen in estrogen receptor-positive breast cancer: a Southwest Oncology Group Study. Clin Cancer Res 4: 7-12.

26. Ravdin PM, Green S, Albain KS (1998) Initial report of the SWOG biologica correlative study of C-erbB-2 expression as a predictor of outcome in a trial comparing adjuvant CAF T with tamoxifen $(\mathrm{T})$ alone. Proc Am Soc Clin Oncol 17: 374

27. Ellis M, Coop A, Singh B (2011) Letrozole is more effective neoadjuvant endocrine therapy than tamoxifen for ErbB-positive breast cancer. J Clin Oncol 19: 3808-16.

28. Interpreting Breast International Group (BIG) 1-98: a randomized, doubleblind, phase III trial comparing letrozole and tamoxifen as adjuvant endocrine therapy for postmenopausal women with hormone receptor-positive, early breast cancer Meredith M Regan, Karen N Price, Anita Giobbie-Hurder, Beat Thürlimann, Richard D Gelber/Breast Cancer Research 2011, 13: 209 http:// breast-cancer-research.com/content/13/3/209

29. Katrine L. Henriksen, Hansen KS, Kirkegaard T, Frogne T (2008) Development of new predictive markers for endocrine therapy and resistance in breast cancer. Acta Oncologic 47: 795-801.

30. Schepotin IB, Zotov AS, Lyubota RV, Anikusko NF, Lyubota II (2013)The basic mechanisms the influence of metabolic syndrome on the risk and prognosis of breast cancer (review). Tumors of female reproductive system 1: 45-51.

31. Fernández-Cuesta L, Oakman C, Falagan-Lotsch P (2012) Prognostic and predictive value of TP53 mutations in node-positive breast cancer patients treated with anthracycline- or anthracycline/taxane-based adjuvant therapy: results from the BIG 02-98 phase III trial. Breast Cancer Res 14.

32. Manié E, Vincent-Salomon A, Lehmann-Che J (2009) High frequency of TP53 mutation in BRCA1 and sporadic basal-like carcinomas but not in BRCA1 luminal breast tumors. Cancer Res, 69: 663- 671.

33. Fernandez-Cuesta L, Anaganti S, Hainaut P (2010) p53 status influences response to tamoxifen but not to fulvestrant in breast cancer cell lines. Int. J. Cancer 128: 1813-21.

34. Kim HS, Yom CK, Kim HJ (2010) Overexpression of p53 is correlated with poor outcome in premenopausal women with breast cancer treated with tamoxifen after chemotherapy. Breast Cancer Res. Treat 12: 777-88.

35. Longley D, Harkin D, Johnston P (2003) 5fluorouracil: mechanisms of action and clinical strategies. Nat Rev Cancer 3: 330-338. 